\section{Prevalência de exposição a ruído ocupacional em trabalhadores brasileiros: resultados da Pesquisa Nacional de Saúde, 2013}

\author{
Prevalence of exposure to occupational noise in \\ Brazilian workers: results of the Brazilian \\ National Health Survey, 2013
}

\section{Prevalencia de exposición al ruido ocupacional de trabajadores brasileños: resultados de la Encuesta Nacional de Salud, 2013}

Ada Ávila Assunção 1

Mery Natali Silva Abreu 1

Priscila Sílvia Nunes Souza 1

\section{Resumo}

O objetivo foi descrever a prevalência de exposição ocupacional a ruído e analisar os fatores associados a essa condição na população brasileira. Neste estudo transversal, de base populacional, utilizaram-se dados da Pesquisa Nacional de Saúde (PNS) de 2013. A amostra foi composta de 36.442 brasileiros com 18 anos ou mais de idade. A variável desfecho foi a exposição autorreferida a ruído durante o trabalho. Para avaliar os possiveis fatores associados à exposição a ruído, foi utilizado o teste qui-quadrado de Pearson, assim como o modelo de Poisson, com variâncias robustas, tanto uni quanto multivariado. Os resultados obtidos identificaram prevalência de exposição ao ruído de 32,1\%, variando de 40,9\% (Santa Catarina) a 21,9\% (Piauí). Houve menor prevalencia entre as mulheres $(R P=0,57$; IC95\%: 0,55-0,59) e no grupo com 55 anos ou mais ( $R P=0,70$; IC95\%: 0,65-0,76). Detectou-se maior prevalência na faixa de 25 a 54 anos ( $R P=1,00$; IC95\%: 0,95-1,06), nível médio de instrução $(R P=1,36$; IC95\%: 1,29-1,44), relato de cansaço $(R P=$ 1,35; IC95\%: 1,30-1,40), dificuldade auditiva ( $R P=1,27 ;$ IC95\%: 1,19-1,35), história de acidente de trabalho $(R P=1,37$; IC95\%: 1,25-1,50). A prevalência de autorrelato de exposição a ruído ocupacional foi superior àquela observada em três países da América Latina. Maior prevalência entre homens, trabalhadores jovens e menos escolarizados era esperada. As associações com problemas auditivos e não auditivos sugerem medidas sistêmicas. Ajustes das perguntas da PNS são desejáveis para favorecer comparabilidade e monitoramento.

Ruído; Inquéritos Epidemiológicos; Exposição Ocupacional; Saúde do Trabalhador

\author{
Correspondência \\ A. A. Assunção \\ Faculdade de Medicina, Universidade Federal de Minas Gerais. \\ Av. Alfredo Balena 190, 6o andar, sala 630, Belo Horizonte, MG \\ 30310-450, Brasil. \\ adavila@medicina.ufmg.br
}

1 Universidade Federal de Minas Gerais, Belo Horizonte, Brasil. 


\section{Introdução}

Ruído é definido como um som indesejado ou uma combinação de diferentes tipos e frequências de som com prováveis efeitos adversos sobre a saúde 1. Ocupa o terceiro lugar no ranking dos riscos ocupacionais classificados pela Organização Mundial da Saúde 2. A exposição ao ruído de alta intensidade e baixa frequência nos locais de trabalho ocorre no país 3 e no mundo 4 , principalmente no setor de transporte, na agricultura, na indústria da transformação e da construção civil, por conta das máquinas (motores, bombas, sistemas de ventilação etc.) ou instalações nesses setores 4,5 . Na indústria da transformação, a exposição ao ruído atinge, no mínimo, $45 \%$ dos empregados 6 .

Alterações das funções físicas e mentais, auditivas ou não, induzidas por esse agente físico configuram um problema de saúde pública reconhecido mundialmente 7 . Em torno de 7 a $21 \%$ do déficit auditivo em trabalhadores estão relacionados a exposição ocupacional ao ruído ${ }^{8}$. Perda auditiva induzida por ruído (Pair) resulta da exposição prolongada. É uma perda auditiva do tipo neurossensorial, geralmente bilateral, irreversível e progressiva com o tempo de exposição (CID 10 - H 83.3) 9. Trauma acústico resulta de um ruído inesperado e intenso, maior que ou igual a 140 decibéis (dB), denominado ruído de impacto, com prováveis danos para o sistema auditivo ${ }^{10}$. Efeitos não auditivos, como dor de cabeça e fadiga ocular 11 ou hipertensão 12, são reconhecidos. Em combinação com prejuízos da audição, as comorbidades são altamente prevalentes, tanto em coortes de idosos quanto de jovens 13 .

A Convenção no 148 da Organização Internacional do Trabalho, aprovada pelo Brasil em 1981, dispõe sobre medidas de controle do ruído ocupacional e indica sanções legais em caso de descumprimento 14 . A legislação trabalhista brasileira estabelece o limite de $85 \mathrm{~dB}(\mathrm{~A})$ para um período de oito horas de exposição contínua ou intermitente ao ruído 15 . Para os ambientes onde as tarefas realizadas exigem atenção e esforço intelectual, o nível aceitável é de até $65 \mathrm{~dB}(\mathrm{~A})$, de maneira a garantir conforto acústico estabelecido pela Norma Brasileira (NBR) no 1015222. A Ordem de Serviço no 6083 determinou às empresas implantar o Programa de Conservação Auditiva (PCA), quando elevado nível de pressão sonora for comprovado.

Os dados sobre a exposição ao ruído ocupacional são escassos 6. Quando disponíveis, referem-se a inquéritos transversais realizados em amostras de trabalhadores formalmente empregados. Mantém-se, assim, desconhecida a situação da população informalmente inserida no mercado de trabalho, geralmente fora tanto do alvo dos programas setoriais quanto das ações sindicais e pesquisas acadêmicas. Falta de informação é uma barreira para o planejamento da atenção à saúde e para efetivar medidas de promoção, de maneira a agravar as iniquidades em saúde 16 . O objetivo foi analisar os fatores associados e descrever a prevalência de exposição ocupacional autorrelatada ao ruído em uma amostra de adultos brasileiros que responderam à Pesquisa Nacional de Saúde (PNS), realizada entre 2013 e 2014

\section{Metodologia}

\section{Fonte dos dados e população alvo}

A PNS é um inquérito populacional desenvolvido por meio da parceria entre Ministério da Saúde, Fundação Oswaldo Cruz (Fiocruz) e Instituto Brasileiro de Geografia e Estatística (IBGE). Como parte do Sistema Integrado de Pesquisas Domiciliares (SIPD) do IBGE, todo o território nacional foi incluído, uma vez dividido em setores censitários da Base Operacional Geográfica de 2010.

A população-alvo da PNS é constituída de pessoas residentes em domicílios particulares permanentes. Foram excluídas da pesquisa as áreas com características especiais e menos populosa. O questionário aplicado pelos técnicos do IBGE nos domicílios selecionados é composto de três partes: informações do domicílio, características gerais de todos os moradores do domicílio e informações de um morador adulto, com 18 anos ou mais de idade, selecionado aleatoriamente. A parte do questionário referente a esse adulto forneceu a maioria das informações de interesse no presente estudo. Obtiveram-se 86\% de taxa de resposta dos moradores selecionados no Brasil 17. 
O projeto PNS foi aprovado pela Comissão Nacional de Ética em Pesquisa (CONEP - Processo: 328.159, em 26 junho de 2013). Todos os dados e informações estão disponíveis no site do IBGE (https://ww2.ibge.gov.br/home/estatistica/populacao/pns/2013/default.shtm).

\section{Planejamento amostral da PNS}

A amostra da PNS é uma subamostra da amostra mestra, que é composta de um conjunto de unidades primárias de amostragem (UPAs). As UPAs são setores censitários ou conjuntos de setores censitários (quando esses setores contam com poucos domicílios). As UPAs foram estratificadas segundo quatro diferentes critérios: administrativo, geográfico, de situação e estatístico. O plano amostral empregado foi o de amostragem por conglomerado em três estágios de seleção, com estratificação das UPAs. No primeiro estágio, a seleção das UPA foi obtida por amostragem aleatória simples entre aquelas previamente escolhidas para a amostra mestra. No segundo estágio, foi selecionado - por amostragem aleatória simples - um número fixo de domicílios particulares permanentes em cada UPA escolhida no primeiro estágio. No terceiro estágio, dentro de cada domicílio da amostra, um morador com 18 anos ou mais de idade foi selecionado - também por amostragem aleatória simples - para responder à terceira parte (individual) do questionário. O tamanho da amostra foi definido, considerando-se o nível de precisão desejado, em intervalos de $95 \%$ de confiança (IC95\%), para as estimativas dos parâmetros de interesse em diferentes níveis de desagregação geográfica e grupos populacionais específicos. O número total de domicílios selecionados foi aproximadamente $23 \%$ maior que a amostra mínima calculada, para levar em consideração a perda total. Em Souza-Júnior et al. 17, estão disponíveis informações detalhadas sobre o desenho amostral da PNS.

A amostra para análise da exposição ao ruído foi constituída de adultos com 18 anos ou mais de idade que responderam à questão E1 do Módulo E: Na semana de 21 a 27 de julho de 2013 (semana de referência), você trabalhou ou estagiou, durante pelo menos uma hora, em alguma atividade remunerada em dinheiro? Considerados ocupados, 36.442 brasileiros que responderam positivamente à E1 constituem a amostra analisada no presente estudo.

\section{A exposição ao ruído}

O Módulo M do questionário da PNS - outras características do trabalho e apoio social - apresenta questões a serem respondidas pelo adulto selecionado entre os moradores do domicílio com 18 anos ou mais de idade. A Questão M11 é desdobrada em oito perguntas atinentes à exposição ocupacional (riscos químicos, físicos e psicossociais), assim definidas: manuseio de substâncias químicas, manuseio de resíduos urbanos (lixo), exposição a ruído (barulho intenso), envolvimento em atividades que levam ao nervosismo, exposição longa ao sol, exposição a material biológico, manuseio de material radioativo e exposição a poeira industrial (pó de mármore).

Considerou-se exposto ao ruído o participante que respondeu positivamente à questão M11b: Pensando em todos os seus trabalhos, o(a) sr(a) está exposto(a) a algum destes fatores que podem afetar a sua saúde? Exposição a ruído (barulho intenso).

\section{Covariáveis}

Em relação aos fatores associados, estudaram-se variáveis sociodemográficas, situação de saúde e comportamentos. Essas variáveis foram classificadas em três blocos distintos. A escolha está embasada no conhecimento atual sobre os efeitos da exposição ao ruído ocupacional. Convém esclarecer, entretanto, a dificuldade em abarcar o conjunto de possíveis efeitos ou situações moduladoras da exposição e associações possíveis. Esse tipo de recorte que restringe a amplitude de hipóteses a serem testadas tem a ver com o desenho transversal do estudo. Apesar desse limite, é possível explorar os resultados produzidos pela primeira vez em âmbito nacional.

Para o primeiro bloco, foram examinados os seguintes fatores: sexo (masculino $\mid$ feminino), faixa etária (18-24 anos | 25-54 anos | 55 anos ou mais), estado civil (com parceiro | sem parceiro), nível de instrução (Ensino Superior | Ensino Médio | Ensino Fundamental ou sem instrução), raça (branca | 
preta | parda | outros). Para o segundo, foram considerados fatores relacionados aos hábitos de vida: consumo de bebida alcoólica uma vez ou mais por mês (não | sim), consumo de álcool três vezes ou mais por semana (não | sim), consumo de risco - heavy episodic drinking - considerado como o consumo de quatro doses ou mais em uma única ocasião (não $\mid$ sim), dirigir sob efeito de álcool (não $\mid$ sim), fumar atualmente (não $\mid \operatorname{sim}$ ), fumou no passado (não $\mid$ sim, diariamente, $\mid$ sim, menos que diariamente), ser fumante passivo (não $\mid$ sim). Por fim, no terceiro bloco, foram analisados os relatos de avaliação de saúde (muito boa | boa | regular, ruim ou muito ruim), problemas de sono (não $\mid$ sim), problema de cansaço (não $\mid$ sim), desinteresse em realizar atividades (não $\mid$ sim), problemas de concentração (não | sim), problemas de alimentação (não $\mid$ sim), lentidão ou agitação ao movimentar-se ou falar (não $\mid$ sim), sentir-se deprimido (não $\mid$ sim), sentir-se mal consigo mesmo (não $\mid$ sim), ideação suicida (não $\mid$ sim), dificuldade auditiva (não $\mid$ sim), uso de aparelho auditivo (não $\mid$ sim), acidente de trabalho nos últimos doze meses (não | sim), deixar de realizar quaisquer de suas atividades habituais devido a acidente de trabalho (não | sim), internação por mais de 24 horas devido a acidente de trabalho (não | sim), sequela e/ou incapacidade decorrente do acidente de trabalho (não | sim), diagnóstico de hipertensão (não | sim), diagnóstico de depressão (não $\mid$ sim).

\section{Análise estatística}

As análises dos resultados sobre a exposição ao ruído foram realizadas com atenção às características da amostra complexa da PNS. Dessa feita, foram considerados os fatores de expansão ou pesos amostrais dos domicílios e todos seus moradores, assim como do morador selecionado para a entrevista. Conforme relatado por Souza-Júnior et al. 17, os pesos dos domicílios e de todos seus moradores foram calculados pelo produto do peso da unidade primária de amostragem correspondente (isto é, ao setor censitário correspondente) e o inverso da probabilidade de seleção do domicílio dentro da unidade primária de amostragem. Os pesos foram ajustados para correção de não respostas e para calibrar as estimativas com os totais populacionais conhecidos de outras fontes. Esses pesos devem ser utilizados na análise das questões referentes às duas primeiras partes do questionário, com informações sobre características dos domicílios e de todos os seus moradores. Os estratos utilizados no plano amostral foram definidos. A expansão amostral foi realizada por meio da utilização do comando svy, disponível no programa Stata (https://www.stata.com), sendo considerada em todas as análises realizadas.

Inicialmente foi realizada uma análise descritiva de todas as variáveis estudadas por meio do cálculo das frequências relativas e elaboração das prevalências, que foram apresentadas no mapa (Figura 1). Todas as análises foram realizadas com o uso do programa Stata 12.0. Para avaliar os possíveis fatores associados à exposição a ruído, foi utilizado o teste qui-quadrado de Pearson, assim como o modelo de Poisson com variâncias robustas, tanto uni quanto multivariado. Para a entrada das variáveis na análise multivariada, foi considerado um valor de p menor que 0,20 na análise univariada. Foram construídos modelos para cada bloco de variáveis (características sociodemográficas, situação de saúde e comportamentos), utilizando o método backward para a retirada das variáveis em cada bloco. Permaneceram no modelo final de cada bloco as variáveis com $\mathrm{p}<0,05$. Em seguida, o modelo final foi ajustado pelas variáveis de todos os blocos, permanecendo somente as significativas no nível de 5\% de significância. Foram estimados os valores de razão de prevalência (RP), com IC95\%, tanto na análise univariada quanto na multivariada. A qualidade do ajuste do modelo foi avaliada por meio da estatística de Deviance. Foram testadas possíveis interações entre as variáveis que permaneceram no modelo final, sendo elas: sexo e idade, sexo e escolaridade, sexo e acidente de trabalho, sexo e relato de cansaço, sexo e dificuldade auditiva. Interações entre idade, escolaridade e demais variáveis supracitadas também foram testadas 


\section{Figura 1}

Prevalência de exposição a ruído ocupacional, de acordo com o estado e Distrito Federal, segundo dados da Pesquisa Nacional de Saúde, Brasil, 2013.

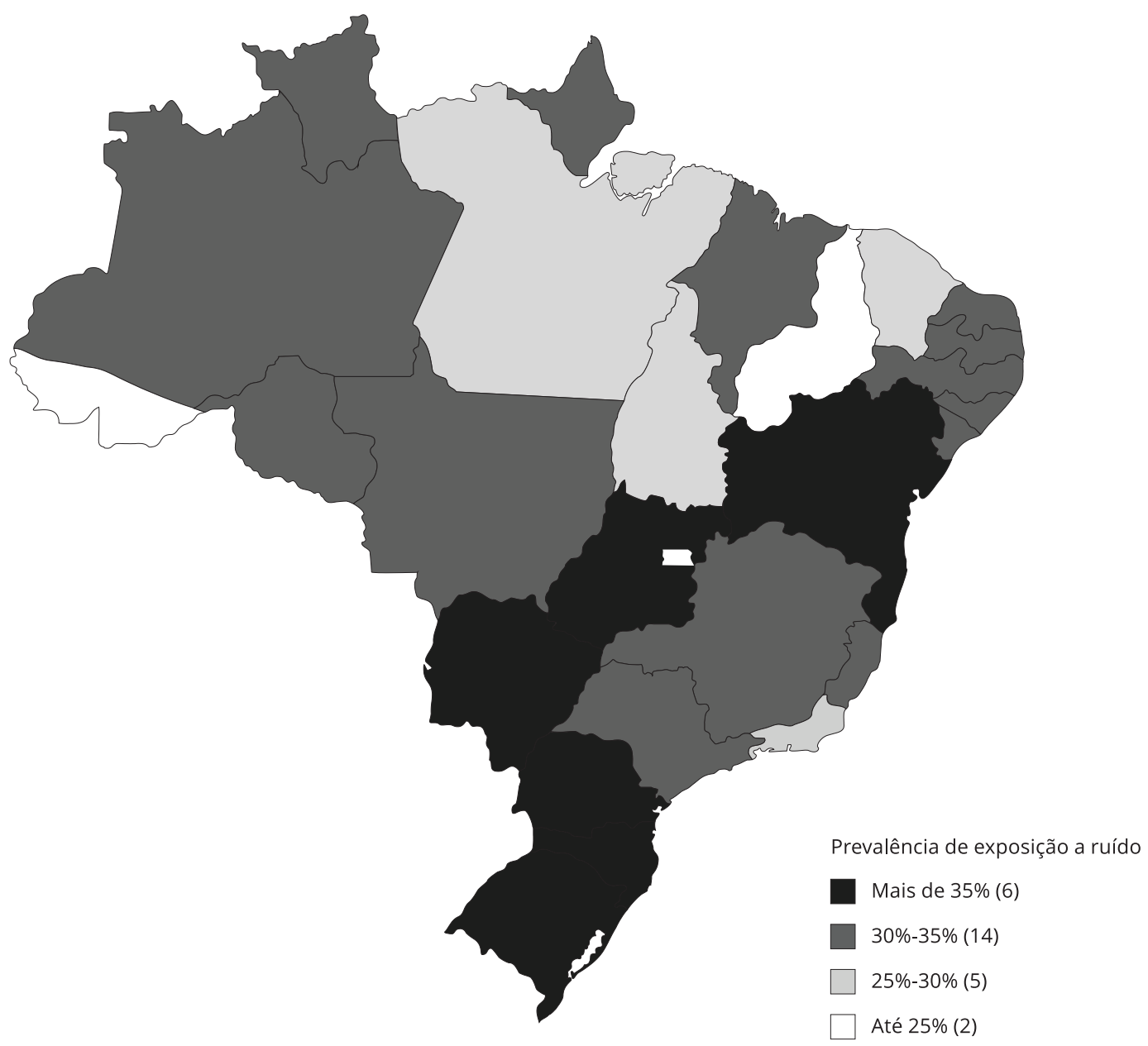

\section{Resultados}

No que se refere à caracterização da amostra estudada, observou-se maioria masculina $(53,4 \%)$, na faixa etária de 25-54 anos (74,5\%), sem união estável (60,2\%). Um total de 41,5\% informou grau de instrução fundamental ou sem instrução e 48,5\% eram pardos (Tabela 1). Um terço dos entrevistados consumia bebida alcoólica uma ou mais vezes no mês, $6,5 \%$ consumiam essa substância três vezes ou mais por semana, 26,3\% dirigiram sob efeito de álcool, 15,4\% fumam atualmente, 84,6\% dos fumantes praticavam o hábito diariamente, 17,2\% eram fumantes passivos (Tabela 2). Quanto à situação de saúde (Tabela 3), 27,8\% autoavaliaram a saúde como regular, ruim ou muito ruim; 27,2\% afirmaram ter problemas de sono; $31 \%$ declararam problemas de cansaço; 21,7\% indicaram desinteresse em realizar atividades; 15,8\% alegaram que possuem problemas de concentração; 16,5\% informaram problemas de alimentação; 12,2\% assumiram lentidão ou agitação ao falar ou movimentar; 19,9\% sentiam-se deprimidos; $11,4 \%$ sentiam-se mal consigo mesmos; $3,1 \%$ pensaram em se ferir ou acharam que seria melhor morrer; 5,9\% foram diagnosticados com depressão e 16,6\% com hipertensão arterial. Enquanto 6,8\% relataram dificuldade para ouvir, 0,1\% utilizam aparelho auditivo, e 2,9\% afirmaram 
Tabela 1

Caracterização e análise univariada dos fatores sociodemográficos associados a exposição a ruído, segundo dados da Pesquisa Nacional de Saúde, Brasil, 2013.

\begin{tabular}{|c|c|c|c|}
\hline & $\begin{array}{l}\text { Percentual da } \\
\text { amostra total } \\
\text { (\%) }\end{array}$ & $\begin{array}{c}\text { Prevalência de } \\
\text { exposição a ruído } \\
\text { (\%) }\end{array}$ & RP (IC95\%) \\
\hline \multicolumn{4}{|l|}{ Sexo } \\
\hline Masculino & 53,4 & 39,7 & 1,00 \\
\hline Feminino & 46,6 & 22,9 & $0,58(0,54-0,61)$ \\
\hline \multicolumn{4}{|l|}{ Faixa etária (anos) } \\
\hline $18-24$ & 12,1 & 33,3 & 1,00 \\
\hline $25-54$ & 74,5 & 33,6 & $1,01(0,93-1,09)$ \\
\hline 55 ou mais & 13,4 & 23,5 & $0,71(0,63-0,77)$ \\
\hline \multicolumn{4}{|l|}{ Estado civil } \\
\hline Com parceiro & 39,8 & 32,4 & 1,00 \\
\hline Sem parceiro & 60,2 & 31,9 & $0,99(0,93-1,04)$ \\
\hline \multicolumn{4}{|l|}{ Nível de instrução } \\
\hline Ensino Superior & 22,5 & 25,7 & 1,00 \\
\hline Ensino Médio & 35,9 & 35,9 & $1,40(1,29-1,51)$ \\
\hline Ensino Fundamental ou sem instrução & 41,5 & 32,2 & $1,25(1,16-1,36)$ \\
\hline \multicolumn{4}{|l|}{ Raça } \\
\hline Branca & 40,5 & 31,2 & 1,00 \\
\hline Preta & 9,5 & 34,7 & $1,11(1,01-1,22)$ \\
\hline Parda & 48,5 & 32,8 & $1,05(0,99-1,12)$ \\
\hline Outros & 1,5 & 27,8 & $0,89(0,71-1,12)$ \\
\hline
\end{tabular}

IC95\%: intervalo de 95\% de confiança; RP: razão de prevalência.

ter sofrido acidente de trabalho. Nesse grupo, 53,2\% deixaram de realizar atividades habituais em decorrência do acidente, 10\% ficaram internados quando sofreram acidente e 18,5\% informaram sequela associada ao sinistro.

A prevalência de exposição a ruído na população brasileira foi de 32,1\% (IC95\%: 31,2\%-33,0\%). No Estado de Santa Catarina, verificou-se a maior prevalência (40,9\%), e no Estado do Piauí, a menor $(21,9 \%)$ (Figura 1), sendo a diferença entre as unidades federativas fortemente significativa $(\mathrm{p}<0,001)$. Em relação à área censitária e região, verificou-se maior prevalência de exposição ao ruído na região Sul-Urbana (37,5\%) e menor na região Norte-Rural (17,4\%) (Figura 1).

Com relação à análise univariada (Tabela 1), as seguintes variáveis foram associadas a maior prevalência de exposição a ruído: ser do sexo masculino, estar na faixa etária de 25-54 anos, ter nível de instrução médio e pertencer à cor/raça preta $(\mathrm{p}<0,05)$.

Os hábitos de vida associados a maior prevalência de exposição a ruído foram: consumo de bebida alcoólica uma vez ou mais por mês, consumo de álcool três vezes ou mais por semana, dirigir sob efeito de álcool, ser fumante atual e fumante passivo (Tabela 2).

Foram significativamente associadas a uma maior prevalência de exposição a ruído as seguintes características relacionadas à saúde: relato de problemas de sono, cansaço, desinteresse, dificuldade de concentração, lentidão ou agitação ao falar ou se movimentar, dificuldade auditiva, problemas alimentares, ter sofrido acidente de trabalho e não ter sido diagnosticado com hipertensão arterial. Sentir-se deprimido ou sentir-se mal consigo mesmo foram respostas associadas a uma maior prevalência de exposição a ruído (Tabela 3).

No modelo final, permaneceram associados a exposição a ruído: sexo, faixa etária, nível de instrução, cansaço, dificuldade auditiva e acidente de trabalho (Tabela 3). A prevalência de exposição a ruído nas mulheres foi 43\% menor ( $\mathrm{RP}=0,57$; IC95\%: 0,55-0,59) que nos homens. Evidenciou-se também 
Tabela 2

Caracterização e análise univariada dos fatores relacionados a hábitos de vida associados a exposição a ruído, segundo dados da Pesquisa Nacional de Saúde, Brasil, 2013.

\begin{tabular}{|c|c|c|c|}
\hline & $\begin{array}{l}\text { Percentual da } \\
\text { amostra total } \\
\text { (\%) }\end{array}$ & $\begin{array}{c}\text { Prevalência de } \\
\text { exposição a ruído } \\
(\%)\end{array}$ & RP (IC95\%) \\
\hline \multicolumn{4}{|c|}{ Consumo de bebida alcoólica 1 vez ou mais por mês } \\
\hline Não & 69,1 & 29,1 & 1,00 \\
\hline Sim & 30,9 & 38,4 & $1,27(1,16-1,39)$ \\
\hline \multicolumn{4}{|c|}{ Consumo de álcool 3 vezes ou mais por semana } \\
\hline Não & 93,5 & 31,5 & 1,00 \\
\hline Sim & 6,5 & 40,0 & $1,27(1,16-1,39)$ \\
\hline \multicolumn{4}{|c|}{ Consumo de risco (heavy episodic drinking) } \\
\hline Não & 43,0 & 37,3 & 1,00 \\
\hline Sim & 57,0 & 39,5 & $1,06(0,98-1,15)$ \\
\hline \multicolumn{4}{|l|}{ Dirigir sob efeito de álcool } \\
\hline Não & 73,7 & 39,6 & 1,00 \\
\hline Sim & 26,3 & 45,2 & $1,14(1,04-1,26)$ \\
\hline \multicolumn{4}{|l|}{ Fumante atual } \\
\hline Não & 84,6 & 31,6 & 1,00 \\
\hline Sim & 15,4 & 35,1 & $1,11(1,03-1,20)$ \\
\hline \multicolumn{4}{|l|}{ Cessação do tabagismo } \\
\hline Não & 84,6 & 30,7 & 1,00 \\
\hline Sim, diariamente & 12,8 & 35,8 & $0,95(0,82-1,10)$ \\
\hline Sim, menos que diariamente & 2,6 & 33,9 & $0,86(0,79-0,92)$ \\
\hline \multicolumn{4}{|l|}{ Fumante passivo } \\
\hline Não & 82,8 & 31,6 & 1,00 \\
\hline Sim & 17,2 & 34,4 & $1,09(1,02-1,17)$ \\
\hline
\end{tabular}

IC95\%: intervalo de 95\% de confiança; RP: razão de prevalência.

\section{Tabela 3}

Caracterização e análise univariada avaliando a situação de saúde associada a exposição a ruído, segundo dados da Pesquisa Nacional de Saúde, Brasil, 2013.

\begin{tabular}{lccc}
\hline & $\begin{array}{c}\text { Percentual da } \\
\text { amostra total } \\
\text { (\%) }\end{array}$ & $\begin{array}{c}\text { Prevalência de } \\
\text { exposição a ruído } \\
\text { (\%) }\end{array}$ & RP (IC95\%) \\
\hline Autoavaliação de saúde & 15,0 & 32,2 & 1,00 \\
$\quad$ Muito boa & 57,3 & 32,1 & $1,00(0,92-1,08)$ \\
Boa & 27,8 & 32,0 & $0,99(0,91-1,08)$ \\
$\quad$ Regular, ruim e muito ruim & 72,8 & 30,9 & 1,00 \\
Problemas de sono & 27,2 & 35,4 & $1,15(1,08-1,22)$ \\
$\quad$ Não & & & 1,00 \\
Sim & 69,0 & 29,8 & $1,25(1,1-1,33)$ \\
Problema de cansaço & 31,0 & 37,4 & \\
$\quad$ Não & & & \\
Sim & & & \\
\hline
\end{tabular}

(continua) 
Tabela 3 (continuação)

\begin{tabular}{|c|c|c|c|}
\hline & $\begin{array}{c}\text { Percentual da } \\
\text { amostra total } \\
\text { (\%) }\end{array}$ & $\begin{array}{c}\text { Prevalência de } \\
\text { exposição a ruído } \\
\text { (\%) }\end{array}$ & RP (IC95\%) \\
\hline \multicolumn{4}{|c|}{ Desinteresse em realizar atividades } \\
\hline Não & 78,3 & 31,2 & 1,00 \\
\hline Sim & 21,7 & 35,8 & $1,15(1,08-1,22)$ \\
\hline \multicolumn{4}{|c|}{ Problemas de concentração } \\
\hline Não & 84,2 & 31,5 & 1,00 \\
\hline Sim & 15,8 & 35,9 & $1,14(1,07-1,22)$ \\
\hline \multicolumn{4}{|c|}{ Problemas de alimentação } \\
\hline Não & 83,5 & 31,5 & 1,00 \\
\hline Sim & 16,5 & 35,4 & $1,12(1,05-1,20)$ \\
\hline \multicolumn{4}{|c|}{ Lentidão/Agitação ao se movimentar/falar } \\
\hline Não & 87,8 & 31,2 & 1,00 \\
\hline Sim & 12,2 & 38,7 & $1,24(1,15-1,33)$ \\
\hline \multicolumn{4}{|c|}{ Sentir-se deprimido } \\
\hline Não & 80,1 & 31,5 & 1,00 \\
\hline Sim & 19,9 & 34,8 & $1,10(1,03-1,18)$ \\
\hline \multicolumn{4}{|c|}{ Sentir-se mal consigo mesmo } \\
\hline Não & 88,6 & 31,7 & 1,00 \\
\hline Sim & 11,4 & 35,5 & $1,12(1,02-1,22)$ \\
\hline \multicolumn{4}{|c|}{ Ideação suicida } \\
\hline Não & 96,9 & 32,1 & 1,00 \\
\hline Sim & 3,1 & 31,5 & $0,98(0,84-1,14)$ \\
\hline \multicolumn{4}{|c|}{ Dificuldade auditiva } \\
\hline Não & 93,2 & 32,3 & 1,00 \\
\hline Sim & 6,8 & 41,7 & $1,36(1,25-1,49)$ \\
\hline \multicolumn{4}{|c|}{ Uso de aparelho auditivo } \\
\hline Não & 99,9 & 32,1 & 1,00 \\
\hline Sim & 0,1 & 23,3 & $0,72(0,31-1,69)$ \\
\hline \multicolumn{4}{|c|}{ Sofreu acidente de trabalho } \\
\hline Não & 97,1 & 31,6 & 1,00 \\
\hline Sim & 2,9 & 49,2 & $1,56(1,39-1,75)$ \\
\hline \multicolumn{4}{|c|}{$\begin{array}{l}\text { Deixou de realizar quaisquer de suas } \\
\text { atividades habituais }\end{array}$} \\
\hline Não & 46,8 & 53,5 & 1,00 \\
\hline Sim & 53,2 & 44,5 & $0,83(0,68-1,03)$ \\
\hline \multicolumn{4}{|c|}{$\begin{array}{l}\text { Internado quando sofreu acidente de } \\
\text { trabalho }\end{array}$} \\
\hline Não & 90 & 49,7 & 1,00 \\
\hline Sim & 10 & 43,7 & $0,88(0,64-1,22)$ \\
\hline \multicolumn{4}{|c|}{ Sequela do acidente de trabalho } \\
\hline Não & 81,5 & 49,3 & 1,00 \\
\hline Sim & 18,5 & 48,9 & $0,99(0,77-1,28)$ \\
\hline \multicolumn{4}{|c|}{ Hipertensão arterial } \\
\hline Não & 83,4 & 32,9 & 1,00 \\
\hline Sim & 16,6 & 28,9 & $0,88(0,82-0,94)$ \\
\hline \multicolumn{4}{|c|}{ Diagnosticado com depressão } \\
\hline Não & 94,1 & 31,1 & 1,00 \\
\hline Sim & 5,9 & 31,9 & $0,99(0,89-1,11)$ \\
\hline
\end{tabular}

IC95\%: intervalo de 95\% de confiança; RP: razão de prevalência. 
prevalência de exposição a ruído 30\% menor entre os indivíduos com 55 anos ou mais $(\mathrm{RP}=0,70$; IC95\%: 0,65-0,76) quando comparados àqueles na faixa etária de 18-24 anos. De um lado, foi observada prevalência 1,36 vez maior no grupo com nível médio de instrução (IC95\%: 1,29-1,44) quando comparado ao grupo com nível de superior de instrução. De outro lado, a prevalência foi 1,14 vez maior no grupo que informou nível fundamental de instrução ou sem instrução (IC95\%: 1,09-1,21) quando comparado ao grupo com nível superior. Cansaço (RP = 1,35; IC95\%: 1,30-1,40), dificuldade auditiva ( $R P=1,27$; IC95\%: 1,19-1,35) e acidente de trabalho ( $R P=1,37$; IC95\%: 1,25-1,50) foram significativamente associados a uma maior probabilidade de exposição a ruído (Tabela 4). Ressalta-se que não foram identificadas interações significativas entre as variáveis que permaneceram no modelo final.

\section{Tabela 4}

Análise multivariada avaliando os fatores sociodemográficos, hábitos de vida e situação de saúde associados a exposição a ruído, segundo dados da Pesquisa Nacional de Saúde, Brasil, 2013.

\begin{tabular}{|c|c|c|c|c|}
\hline & \multicolumn{4}{|c|}{ RP (IC95\%) } \\
\hline & $\begin{array}{l}\text { Modelo Bloco 1: } \\
\text { variáveis sociodemográficas }\end{array}$ & $\begin{array}{l}\text { Modelo Bloco 2: } \\
\text { hábitos de vida }\end{array}$ & $\begin{array}{l}\text { Modelo Bloco 3: } \\
\text { situação de saúde }\end{array}$ & Modelo final \\
\hline \multicolumn{5}{|l|}{ Sexo } \\
\hline Masculino & 1,00 & & & 1,00 \\
\hline Feminino & $0,58(0,54-0,61)$ & & & $0,57(0,55-0,59)$ \\
\hline \multicolumn{5}{|l|}{ Faixa etária (anos) } \\
\hline $18-24$ & 1,00 & & & 1,00 \\
\hline $25-54$ & $1,07(0,99-1,16)$ & & & $1,00(0,95-1,06)$ \\
\hline 55 ou mais & $0,74(0,65-0,83)$ & & & $0,70(0,65-0,76)$ \\
\hline \multicolumn{5}{|l|}{ Nível de instrução } \\
\hline Ensino Superior & 1,00 & & & 1,00 \\
\hline Ensino Médio & $1,33(1,19-1,49)$ & & & $1,36(1,29-1,44)$ \\
\hline Ensino Fundamental ou sem instrução & $1,24(1,11-1,38)$ & & & $1,14(1,09-1,21)$ \\
\hline \multicolumn{5}{|l|}{ Dirigir sob efeito de álcool } \\
\hline Não & & 1,00 & & - \\
\hline $\operatorname{Sim}$ & & $1,14(1,04-1,26)$ & & - \\
\hline \multicolumn{5}{|l|}{ Lentidão/Agitação ao se movimentar/falar } \\
\hline Não & & & 1,00 & - \\
\hline Sim & & & $1,10(1,02-1,19)$ & - \\
\hline \multicolumn{5}{|l|}{ Problema de cansaço } \\
\hline Não & & & 1,00 & 1,00 \\
\hline Sim & & & $1,19(1,12-1,27)$ & $1,35(1,30-1,40)$ \\
\hline \multicolumn{5}{|l|}{ Dificuldade auditiva } \\
\hline Não & & & 1,00 & 1,00 \\
\hline Sim & & & $1,29(1,18-1,41)$ & $1,27(1,19-1,35)$ \\
\hline \multicolumn{5}{|l|}{ Acidente de trabalho } \\
\hline Não & & & 1,00 & 1,00 \\
\hline Sim & & & $1,49(1,33-1,68)$ & $1,37(1,25-1,50)$ \\
\hline
\end{tabular}

IC95\%: intervalo de 95\% de confiança; RP: razão de prevalência.

Nota: valor de $\mathrm{p}$ ajuste do modelo final $=1,000$. 


\section{Discussão}

Este estudo representativo da população brasileira avaliou, em caráter inédito, a exposição a ruído ocupacional por meio de autorrelato de sujeitos (18 anos ou mais) que estavam trabalhando ou afastados do trabalho na semana de referência da entrevista domiciliar. Evidenciaram-se associações com problemas auditivos e não auditivos, embora surpreendentemente não tenha sido verificada associação com hipertensão, nem com tabagismo.

A prevalência encontrada, $32,1 \%$, foi maior quando comparada àquela obtida em inquéritos populacionais de outros países latino-americanos: 25,8\%, 20,5\% e 16,7\% em El Salvador, Costa Rica e Argentina, respectivamente 18 . Ainda que comparações sejam limitadas por conta da falta de consenso metodológico 19 , os resultados descritos são preocupantes diante da estimativa de $16 \%$ a $24 \%$ de problemas auditivos relacionados a exposição ao ruído, que é responsável por mais de quatro milhões de anos de vida perdidos ou vividos com incapacidade (DALYs) 8 .

Em convergência com os resultados dos outros países, observou-se maior prevalência entre os menos escolarizados 20 quando comparados aos mais escolarizados, e entre os homens 5,7 quando comparados às mulheres. Esse resultado é convergente: os menos escolarizados são mais expostos aos riscos ocupacionais em geral, porque postos de trabalho com mais exposição ao ruído são aqueles dos setores da economia com menor exigência de instrução para desenvolver as tarefas 21 .

Para interpretar a maior prevalência entre os homens, convém levar em conta a divisão sexual no trabalho em nossa sociedade, ou seja, homens e mulheres, quando inseridos no mercado de trabalho, são distribuídos de forma heterogênea entre os setores da produção 21. Enquanto os homens são majoritários na construção civil, no setor extrativista e na indústria de transformação, que concentram inúmeras fontes de ruído, as mulheres predominam na indústria microeletrônica, no telemarketing e no setor educacional 22, que são setores cujos ambientes sonoros se caracterizam por ruídos de menor intensidade. A inserção na força de trabalho apresenta outros recortes de gênero, pois, em um mesmo setor ou empresa, homens e mulheres não executam tarefas similares, resultando em diferenciais de exposição. Assim se explica a maior concentração de mulheres em tarefas com exigência de esforço ou posturas estáticas e movimentos repetitivos, ao passo que os homens são mais expostos a ruído do que elas, provocado tanto por ferramentas quanto por máquinas e instalações diversas 23 .

A prevalência da exposição foco foi maior no grupo dos mais jovens, provavelmente em função da distribuição da força de trabalho, de acordo com a faixa etária, nos diferentes ramos da produção. Contudo, é também provável que os mais velhos, mais sensíveis ao ruído ambiental, em função dos efeitos da presbiacusia, tenham evitado ou não suportado ambientes barulhentos 24 . Ou então, seria resultado da rígida seleção ao exame admissional nas empresas, uma vez que as audiometrias entre os exames preconizados teriam eliminado a entrada daqueles com alterações no ouvido interno, mais provavelmente entre os mais velhos.

A associação com dificuldade auditiva e com cansaço era esperada. Os danos auditivos são assintomáticos nas fases iniciais. Os sintomas se manifestam anos depois, a depender da intensidade e da duração da exposição. Os danos têm relação direta com intensidade, duração, tipo e frequência do ruído. No setor de serviços administrativos, $35 \%-40 \%$ dos trabalhadores estão muito irritados com níveis de ruído entre $55 \mathrm{~dB}$ e $60 \mathrm{~dB}(\mathrm{~A}) 25$. Cansaço é uma expressão da situação em que se acumulam reações subjetivas (medo, tristeza, isolamento social) e físicas (alterações cardiovasculares, dor de cabeça, fadiga visual). No conjunto, essas reações repercutem negativamente sobre o ciclo sono-vigília e explicam a redução do desempenho cognitivo que se manifesta em déficit da atenção, memória e vigília. Essas últimas, por sua vez, estão na origem de acidentes de trabalho 11,26. Em uma região industrial do Brasil, o risco relativo de acidentar-se foi 3,7 e 5 vezes maior quando o trabalhador informou estar, às vezes ou sempre, respectivamente, exposto a ruído 27.

A exposição ao ruído sofre variações, pois os trabalhadores realizam diferentes tarefas durante a jornada, além de estarem expostos ao ruído produzido nos postos de trabalho dos colegas. É necessário levar em conta a suscetibilidade individual. Vale ainda ressaltar a exposição não ocupacional, de maneira a assumir a necessidade de uma abordagem global quando o objetivo é a prevenção 22 .

O relato de ter sido diagnosticado com hipertensão arterial, surpreendentemente, não foi associado a exposição a ruído ocupacional, cujo efeito sobre o aparelho cardiovascular está bem documentado 13. Efeitos crônicos da exposição de longo prazo ao ruído, incluindo aqueles de natureza cardiovas- 
cular, além de inespecíficos, são multifatoriais. Estudos transversais são limitados para elucidar essa dinâmica, porque são realizados em um corte temporal, em que exposição e dano são investigados simultaneamente. Além disso, as pessoas intervêm em suas próprias situações, por exemplo, mudando de emprego ou mudando de ambientes mais ruidosos para lugares mais silenciosos. Embora se reconheça a vantagem da PNS de evitar a sub-representação dos expostos, como é o caso dos estudos que investigam exclusivamente os sujeitos com vínculo formal de emprego, os seus resultados não são potentes para capturar a dinâmica mencionada.

Está suficientemente validado o uso de dados autorrelatados sobre exposição ao ruído 28 . A correlação entre exposição percebida e medida por dosimetria encontra-se bem documentada 29,30. Recentemente, foram comparados resultados das mensurações por meio de equipamentos e autopercepção do ruído ambiental. Quanto a essa última, o questionário apresentou três perguntas. A primeira inquiria sobre a dificuldade em ouvir os outros por causa do ruído no ambiente; a segunda, se era necessário gritar para que os outros o escutassem; e a terceira versava sobre a frequência da exposição. Observou-se relação linear em cada um dos itens autopercebidos e as mensurações por dosimetria, que foram realizadas paralelamente pelos pesquisadores 31 .

A pergunta clássica utilizada nos inquéritos é a necessidade de elevar a voz para conversar com outra pessoa no ambiente laboral 19. A resposta positiva é considerada proxy para nível sonoro superior a $80-85 \mathrm{dBA} 32$. Diferentemente de inquéritos que, além da referida pergunta, introduzem questões para avaliar a duração e intensidade de exposição 33 , na PNS, a única pergunta nesse quesito indaga se o adulto está ou não exposto.

Sinalizadas as dificuldades para comparar, convém ressaltar o ineditismo da pesquisa que, além de extrapolar setores específicos, abrangeu afastados do trabalho, mulheres e homens, independentemente do tipo de vínculo empregatício e da faixa etária acima dos 18 anos. Por fim, é representativa para a população brasileira.

Os problemas auditivos relacionados a exposição ao ruído ocupacional permanecem um dilema que justifica a extensa pesquisa no tema, ainda que propalados os padrões tanto para ações no âmbito da engenharia de produção quanto para aqueles referentes à conservação auditiva 7 . Existem avanços na compreensão sobre os mecanismos moleculares dos danos às células e aos nervos auditivos. Há expectativas terapêuticas indicadas pelas pesquisas recentes que usaram células-tronco para recuperar o dano auditivo sensorial. Evidências orientam diretrizes para prevenção calcada nos efeitos da interação de diferentes fontes, características individuais, exposição concomitante a substâncias químicas e exposição durante o tempo de lazer. No conjunto, as inovações em curso são animadoras, mas permanecem os desafios relacionados à elevada proporção de trabalhadores brasileiros expostos, conforme descrito acima.

A PNS é uma oportunidade para a saúde do trabalhador, sobretudo porque inclui o conjunto da força de trabalho. Está reconhecida a necessidade de ultrapassar a barreira da informação quando as únicas fontes de dados disponíveis são a Previdência Social ou os resultados de estudos focalizados em uma categoria de trabalhadores presentes no estabelecimento, no momento da entrevista. Quanto aos dados previdenciários, as informações dizem respeito unicamente ao grupo dos trabalhadores formalmente vinculados ao emprego, que representem em média, no país, $50 \%$ a $60 \%$ da força de trabalho. Mudanças recentes vão acirrar essa situação, na medida em que o avanço da desregulamentação trabalhista tende a provocar ampliação do contingente de subempregados 19. Em relação aos inquéritos ocupacionais, sabe-se das iniquidades na distribuição dos estudos realizados no Brasil, tanto setorial quanto regionalmente 34 . Nesse contexto, os resultados da PNS abrem vias para resolver os impasses mencionados diante de informações exclusivamente relacionadas à força de trabalho vinculada formalmente ao emprego e/ou concentrada em um setor, cidade ou região do país. Não obstante, existem limites a serem superados. Conforme mencionado, a pergunta do questionário não assegura comparabilidade e monitoramento. Além dessa imperfeição, viés de informação é esperado, haja vista as restrições de se obter informações sobre tipo, duração e frequência da exposição por meio de uma única pergunta. É esperada subestimação dos resultados sobre morbidades quando eles foram obtidos por autorrelato, uma vez conhecidos vieses derivados de problemas de memória, barreiras de linguagem e representações sociais nos inquéritos de saúde 35. Estudos anteriores, entretanto, confirmaram a validade da autorreferência sobre deficiência auditiva. Os autores encontraram alta sensibilidade (74\%) e alta especificidade (72\%) quando utilizada apenas uma pergunta sobre essa condição 36. Em 
vez de "deficiência”, na PNS, o substantivo utilizado é "dificuldade". Seria o caso de provocar mudança nos termos utilizados nas próximas edições da pesquisa nacional? A opção de manter a classificação do IBGE foi vantajosa, haja vista a expertise desse Instituto e capacidade comprovada de realizar e publicar periodicamente, há mais de 30 anos, resultados de pesquisas nacionais. Entretanto, a terminologia do IBGE para classificar a população ocupada, apesar de representar um esforço concatenado às diretrizes da OIT, ainda não é potente o suficiente para capturar e sistematizar as diferentes modalidades de inserção na força de trabalho.

Os resultados descritos são representativos da população brasileira. Eles expressam uma complexidade a merecer o desenvolvimento de proposições para abarcar os níveis micro, meso e macro da exposição ao ruído ocupacional e problemas de saúde associados. Sintomas auditivos e não auditivos indicam reforçar a proposição de estratégia sistêmica, a fim de apoiar medidas de prevenção, diagnóstico precoce e monitoramento. Foco no indivíduo exposto (nível micro), sintomático ou não, ativo ou incapacitados, é relevante, conforme pretendido no Programa de Conservação Auditiva 3 adotado em empresas. O Protocolo Pair do Ministério da Saúde 9 instrui os profissionais do Sistema Único de Saúde sobre os parâmetros para diagnóstico, tratamento e prevenção, além de procedimentos a serem desenvolvidos desde o primeiro atendimento até a notificação. A Rede de Atenção à Saúde Auditiva 37 do Ministério da Saúde estimula a criação de programas de vigilância ocupacional amparados por equipes multiprofissionais, bem como diretrizes para reabilitação e reinserção dos trabalhadores atingidos. Concepção ergonômica (nível meso), tanto das plantas das indústrias quanto do design de equipamentos e instalações, é fortemente recomendada para eliminar ou atenuar o risco 7 . Políticas setoriais 38 (nível macro) de promoção do ambiente de trabalho seguro, combinadas às estratégias de formação do trabalhador, além de educação de qualidade e em quantidade para garantir o acesso universal em todo território nacional, seriam frutíferas a longo prazo.

\section{Conclusão}

Apesar da existência de um marco regulatório multissetorial, específico e abrangente, este estudo representativo da população nacional identificou alta prevalência de exposição a ruído no ambiente de trabalho dos adultos ocupados em 2013. Queixas auditivas e extra-auditivas foram associadas à exposição investigada, conforme esperado. Associações com acidentes de trabalho e maior prevalência entre os mais jovens indicam a vulnerabilidade do grupo exposto quanto à necessária capacidade funcional para manter-se no emprego. Medidas de monitoramento mais eficazes dos ambientes ocupacionais são necessárias. 


\section{Colaboradores}

A. A. Assunção foi responsável pela concepção do artigo, revisou a literatura, redigiu a primeira versão e aprovou a versão final para publicação. M. N. S. Abreu analisou os dados, colaborou na redação do artigo, participou da revisão final e aprovação para publicação. P. S. N. Souza participou da análise dos dados e revisou a versão final para publicação.

\section{Informações adicionais}

ORCID: Ada Ávila Assunção (0000-0003-21230422); Mery Natali Silva Abreu (0000-0002-66913537); Priscila Sílvia Nunes Souza (0000-00030203-6034).

\section{Agradecimentos}

Ao Conselho Nacional de Desenvolvimento Científico e Tecnológico (CNPq).

\section{Referências}

1. Seidman MD, Standring RT. Noise and quality of life. Int J Environ Res Public Health 2010; 7:3730-8.

2. World Health Organization. Global health risks: mortality and burden of disease attributable to selected major risks. Geneva: World Health Organization; 2009.

3. Ordem de Serviço INSS/DAF/DSS no 608. http://www.oficionet.com.br/arquivos_links/ INSS/OS608-INSS-05-08-98.pdf (acessado em 25/Mar/2018).

4. Pawlaczyk-Luszczyńiska M, Dudarewicz A, Waszkowska M, Szymczak W, SliwińskaKowalska M. The impact of low-frequency noise on human mental performance. Int J Occup Med Environ Health 2005; 18:185-98.

5. Meira TC, Santana VS, Ferrite S. Gênero e fatores associados ao uso de equipamentos de proteção auditiva no trabalho. Rev Saúde Pública 2015; 49:76.

6. Cavalcante F, Ferrite S, Meira TC. Exposição ao ruído na indústria de transformação no Brasil. Rev CEFAC 2013; 15:1364-70.

7. Basner M, Babisch W, Davis A, Brink M, Clark C, Janssen S, et al. Auditory and non-auditory effects of noise on health. Lancet 2014; 383:1325-32.

8. Nelson DI, Nelson RY, Concha-Barrientos M, Fingerhut M. The global burden of occupational noise-induced hearing loss. Am J Ind Med 2005; 48:446-58.

9. Departamento de Ações Programáticas Estratégicas, Secretaria de Atenção à Saúde, Ministério da Saúde. Perda auditiva induzida por ruído (Pair). Brasília: Ministério da Saúde; 2006.

10. Yehudai N, Fink N, Shpriz M, Marom T. Acute acoustic trauma among soldiers during an intense combat. J Am Acad Audiol 2017; 28:43643

11. Kim J, Lee W, Won JU, Yoon JH, Seok H, Kim Yk, et al. The relationship between occupational noise and vibration exposure and headache/eyestrain, based on the fourth Korean Working Condition Survey (KWCS). PLoS One 2017; 12:e0117846.

12. Chang TY, Hwang BF, Liu CS, Chen RY, Wang VS, Bao BY, et al. Occupational noise exposure and incident hypertension in men: a prospective cohort study. Am J Epidemiol 2013; 177:818-25.

13. Stam M, Kostense PJ, Lemke U, Merkus P, Smit JH, Festen JM, et al. Comorbidity in adults with hearing difficulties: which chronic medical conditions are related to hearing impairment? Int J Audiol 2014; 53:392-401.

14. Brasil. Decreto no 93.413 de 15 de outubro de 1986. Promulga a Convenção no 148 sobre a Proteção dos Trabalhadores Contra os Riscos Profissionais Devidos à Contaminação do Ar, ao Ruído e às Vibrações no Local de Trabalho. Diário Oficial da União 1986; 16 out. 
15. Ministério do Trabalho e Emprego. Portaria no 1.297, de 13 de agosto de 2014. Aprova o Anexo 1 - Vibração - da Norma Regulamentadora no 9 - Programas de Prevenção de Riscos Ambientais (PPRA), altera o Anexo 8 - Vibração - da Norma Regulamentadora no 15 - Atividades e Operações Insalubres, e dá outras providências. Diário Oficial da União 2014; 14 ago.

16. Bambra C. Work, worklessness and the political economy of health inequalities. J Epidemiol Community Health 2011; 65:746-50.

17. Souza-Júnior PRB, Freitas MPS, Antonaci GA, Szwarcwald CL. Desenho da amostra da Pesquisa Nacional de Saúde 2013. Epidemiol Serv Saúde 2015; 24:207-16.

18. Benavides FG, López-Ruiz M, Rojas M, Herrera MSP, Chavarría J, Cornelio C, et al. Informe de salud ocupacional en América Latina y el Caribe, brechas de desigualdad en la salud de las personas que trabajan. Heredia: Programa Salud, Trabajo y Ambiente, Instituto Regional de Estudios en Sustancias Tóxicas, Universidad Nacional de Costa Rica; 2018.

19. Benavides FG, Merino-Salazar PM, Cornelio C, Assunção AA, Agudelo-Suárez AA, Amable $\mathrm{M}$, et al. Cuestionario básico y criterios metodológicos para las Encuestas sobre Condiciones de Trabajo, Empleo y Salud en América Latina y el Caribe. Cad Saúde Pública 2016; 32:e00210715.

20. Furnée CA, Groot W, van den Brink HM. The health effects of education: a meta-analysis. Eur J Public Health 2008; 18:417-21.

21. Eng A, 't Mannetje A, McLean D, Ellison-Loschmann L, Cheng S, Pearce N. Gender differences in occupational exposure patterns. Occup Environ Med 2011; 68:888-94.

22. Feder K, Michaud D, McNamee J, Fitzpatrick E, Davies H, Leroux TJ. Prevalence of hazardous occupational noise exposure, hearing loss, and hearing protection usage among a representative sample of working Canadians. J Occup Environ Med 2017; 59:92-113.

23. Messing K, Mager Stellman J. Sex, gender and women's occupational health: the importance of considering mechanism. Environ Res 2006; 101:149-62.

24. Maccà I, Scapellato ML, Carrieri M, Maso S, Trevisan A, Bartolucci GB. High-frequency hearing thresholds: effects of age, occupational ultrasound and noise exposure. Int Arch Occup Environ Health 2015; 88:197-211.

25. Gourévitch B, Edeline JM, Occelli F, Eggermont JJ. Is the din really harmless? long-term effects of non-traumatic noise on the adult auditory system. Nat Rev Neurosci 2014; 15:48391.

26. Yoon JH, Roh J, Kim CN, Won JU. The risk of occupational injury increased according to severity of noise exposure after controlling for occupational environment status in Korea. Noise Health 2016; 18:355-61.
27. Cordeiro R, Clemente APG, Diniz CS, Dias A. Exposição ao ruído ocupacional como fator de risco para acidentes do trabalho. Rev Saúde Pública 2005; 39:461-6.

28. Schlaefer K, Schlehofer B, Schüz J. Validity of self-reported occupational noise exposure. Eur J Epidemiol 2009; 24:469-75.

29. Hagerman B. Musicians' ability to judge the risk of acquiring noise induced hearing loss. Noise Health 2013; 15:199-203.

30. Neitzel RL, Svensson EB, Sayler SK, AnnChristin J. A comparison of occupational and nonoccupational noise exposures in Sweden. Noise Health 2014; 16:270-8.

31. Neitzel RL, Andersson M, Andersson E. Comparison of multiple measures of noise exposure in paper mills. Ann Occup Hyg 2016; 60:581-96.

32. Dzhambov AM, Dimitrova DD, Tokmakov MP. Association between self-reported occupational noise and the prevalence of stroke: secondary analysis of the National Health Interview Survey, 2014. Noise Control Eng J 2016; 64:779-88.

33. Dzhambov AM, Dimitrova DD. Long-term self-reported exposure to occupational noise is associated with BMI-defined obesity in the US general population. Am J Ind Med 2016; 59:1009-19.

34. Gobbi T, Lima EP, Assunção AA. Panorama dos inquéritos ocupacionais no Brasil (20052015): uma revisão sistemática da literatura. Ciênc Saúde Colet 2018; 24:2923-32.

35. Paiva KM, Cesar CLG, Alves MCGP, Barros MBA, Carandina L, Goldbaum M. Envelhecimento e deficiência auditiva referida: um estudo de base populacional. Cad Saúde Pública 2011; 27:1292-300.

36. Torre P, Moyer CJ, Haro NR. The accuracy of self-reported hearing loss in older LatinoAmerican adults. Int J Audiol 2006; 45:559-62.

37. Ministério da Saúde. Portaria no 587 de 7 de outubro de 2004. 1. Ministério da Saúde. Portaria no 587, de 07 de outubro de 2004. Determina que as Secretarias de Estado da Saúde dos estados adotem as providências necessárias à organização e implantação das Redes Estaduais de Atenção à Saúde Auditiva e dá outras providências. Diário Oficial da União 2004; 11 out.

38. Brasil. Decreto no 7.602 , de 7 de novembro de 2011. Dispõe sobre a Política Nacional de Segurança e Saúde no Trabalho. Diário Oficial da União 2011; 8 out. 


\section{Abstract}

The objectives were to describe the prevalence of occupational exposure to noise and to analyze associated factors in the Brazilian population. This cross-sectional population-based study used data from the Brazilian National Health Survey (PNS, 2013). The sample consisted of 36,442 Brazilians 18 years or older. The outcome variable was self-reported exposure to noise during work. To assess possible factors associated with exposure to noise, we used Pearson's chi-square test and the univariate and multivariate Poisson model with robust variance. The results showed $32.1 \%$ prevalence of exposure to noise, varying from $40.9 \%$ (Santa Catarina, Brazil) to 21.9\% (Piaui, Brazil). Prevalence was lower in women $(P R=0.57$; 95\% CI: 0.55-0.59) and in individuals 55 years or older $(P R=0.70 ; 95 \% \mathrm{CI}: 0.65-0.76)$. Higher prevalence was associated with the 25-54-year age bracket $(P R=1.00$; 95\%CI: 0.95-1.06), middle schooling level $(P R=1.36$; 95\%CI: 1.29-1.44), self-reported fatigue $(P R=1.35 ; 95 \% C I: 1.30$ 1.40), hearing impairment $(P R=1.27 ; 95 \% C I$ : 1.19-1.35), and history of work-related accident $(P R=1.37 ; 95 \% C I: 1.25-1.50)$. Prevalence of selfreported exposure to occupational noise was higher than in three other Latin American countries. Higher prevalence among men, younger workers, and those with less schooling was expected. The associations with auditory and non-auditory problems suggest the need for systemic interventions. Adjustments to the questions in the PNS are desirable to favor comparability and monitoring.

Noise; Health Surveys; Occupational Exposure; Occupational Health

\section{Resumen}

El objetivo fue describir la prevalencia de exposición ocupacional al ruido y analizar factores asociados a esta condición en la población brasileña. En este estudio transversal, de base poblacional, se utilizaron datos de la Encuesta Nacional de Salud (PNS) de 2013. La muestra estaba compuesta por 36.442 brasileños con 18 años o más de edad. La variable desenlace fue la exposición autoinformada al ruido durante el trabajo. Para evaluar los posibles factores asociados a la exposición al ruido se utilizó el test chi-cuadrado de Pearson, así como el modelo de Poisson con variancias robustas, tanto uni-como multivariado. Los resultados obtenidos identificaron una prevalencia de exposición al ruido de 32,1\%, variando de 40,9\% (Santa Catarina) a 21,9\% (Piauí). Menor prevalencia entre las mujeres ( $R P=0,57$; IC95\%: 0,55-0,59) y en el grupo con 55 años o más ( $R P=0,70$; IC95\%: 0,650,76). Mayor prevalencia en la franja de 25 a 54 años ( $R P=1,00$; IC95\%: 0,95-1,06), nivel medio de instrucción $(R P=1,36$; IC95\%: 1,29-1,44), relatos de cansancio $(R P=1,35$; IC95\%: 1,30-1,40), dificultad auditiva ( $R P=1,27 ;$ IC95\%: 1,19-1,35), historia de accidente laboral $(R P=1,37$; IC95\%: 1,25-1,50). La prevalencia de autoinforme de exposición a ruido ocupacional fue superior a la observada en tres países latinoamericanos. La mayor prevalencia entre hombres, trabajadores jóvenes y menos escolarizados era esperada. Las asociaciones con problemas auditivos y no auditivos sugieren medidas sistémicas. Es deseable que se produzcan ajustes en las preguntas de la PNS para favorecer comparabilidad y el monitoreo.

Ruido; Encuesta Epidemiológicas; Exposición

Profesional; Salud Laboral
Recebido em 13/Mai/2018

Versão final reapresentada em 20/Mar/2019

Aprovado em 18/Abr/2019 